DEUTSCHE AKADEMIE DER WISSENSCHAFTEN ZU BERLIN SCHRIFTEN DES INSTITUTS FÜR GESCHICHTE REIHE I: ALLGEMEINE UND DEUTSCHE GESCHICHTE BAND 2

JÜRGEN KUCZYNSKI

\title{
STUDIEN ZUR GESCHICHTE DES KAPITALISMUS
}

AKA DEMIE-VERLA G B E R I N 1957 
Copyright 1957 by Akademie-Verlag GmbH., Berlin Alle Rechte vorbehalten Erschienen im Akademie-Verlag GmbH., Berlin W 8, Mohrenstraße 39 Lizenz-Nr. 202 • ${ }^{-}$100/625/56 Satz: Verlag Junge Welt, Berlin

Druck und Bindung: Druckhaus "Maxim Gork1", Altenburg

Bestell- und Verlagsnummer: 2083/I/2

Printed in Germany 